\title{
Consultation liaison psychiatry for COVID-19 inpatients: A novel care delivery program in Morocco
}

\author{
Roukaya Benjelloun ${ }^{1} \cdot$ Yassine Otheman $^{2}$ (D) $\cdot$ Chafik El Kettani $^{1}$ \\ Accepted: 13 November 2020 / Published online: 16 November 2020 \\ (C) Springer Science+Business Media, LLC, part of Springer Nature 2020
}

\section{Introduction}

Since its onset in late 2019, the COVID-19 outbreak has become a worldwide medical crisis, declared as a public health emergency of international concern by the World Health Organization (WHO) on January 30th, 2020 (Wang et al., 2019). Mental health is considered a major issue during this health worldwide crisis (Asdmunson, 2020); while data on mental health issues in COVID-19 inpatients are more and more available (Hu et al., 2020). Here we report results of a novel care delivery program in the current context of COVID19 outbreak, in Cheikh Khalifa University Hospital in Casablanca, Morocco.

\section{Description of the Mental Health Circuit for COVID-19 Inpatients}

We set up protocols and specific tools to implement a psychiatric support system facilitating the intervention of physicians assigned to the COVID-19 ward:

\section{Information Sheet for Patients and Psychotropic Drug Protocol for Physicians}

Noticing that patient's distress was considerably fueled by the ignorance of « what was going on for them », we elaborated an information sheet that contains specific information related to the COVID-19 unit routines as well as WHO recommendations about sleep and stress management (https://www.who.int/

Yassine Otheman

yassine.othemane@usmba.ac.ma

1 Faculty of Medicine, Mohammed VI University of Health Sciences (UM6SS), Casablanca, Morocco

2 Faculty of Medicine and Pharmacy, Sidi Mohamed Ben Abdellah University, Fez, Morocco
publications-detail/WHO-2019-nCoV-MentalHealth-2020.1, n.d.). We also elaborated guidelines for managing acute insomnia, anxiety, and psychiatric agitation for the first line physicians, looking after the COVID-19 ward. We mentioned drugs to avoid regarding their interactions with hydroxy-chloroquine and azithromycine association, recommended anxiolytics and hypnotics with short half-lives, at lower doses and for short durations and we conditioned the prescription of antipsychotics by a previous cardiac assessment.

\section{Psychiatric Interventions for COVID-19 Inpatients}

Depending on the severity of their initial symptoms, patients with COVID-19 were admitted either in regular rooms, intensive care unit or resuscitation ward. We systematically assessed patients initially admitted in regular rooms or intensive care unit within two days following their admission. Further assessments by telephone were scheduled for symptomatic patients for whom we indicated a specific intervention, at patients' demand or when medical staff noticed that patients showed symptoms of psychological distress. The psychiatric evaluation of patients initially admitted in resuscitation ward was previously conditioned by the authorization of the resuscitation staff following a case by case assessment.

We decided to run our first interviews face to face for two main reasons: First, because phone assessment did not enable us to evaluate para-verbal expression of potential psychiatric symptoms, and second, it seemed important for us to meet patients for at least once in person, to humanize the first contact and build therapeutic alliance with them.

Before getting in COVID-19 wards, we had to apply hospital guidelines regarding safety measures and go through the following steps: Follow the specific COVID-19 circuit (dedicated elevator and staircases); get equipped with protective clothing, surgical gloves, shoe covers, headgears, face shields and FFP2 face masks. Once inside the patient rooms, we asked patients to put on their face mask and open the window. We kept at least two meters far from patients and we avoided touching walls or furniture. Nothing could get in and then out 
from the patient room. Therefore, we did not take note during interviews.

The initial face-to-face interview lasted fifteen to thirty minutes and included the assessment of psychiatric history, circumstances in which COVID-19 was diagnosed, whether the patient had family members affected by or deceased due to COVID-19, quality and duration of sleep and symptoms of anxiety, depression and acute stress.

We had to constantly adapt our interventions, duration of interview, drug doses, and rhythm of follow up to the patient's clinical condition. Pharmacotherapy consisted of short-term prescription of three main drugs (at their lower doses): Lorazepam for anxiety symptoms, Zolpidem for insomnia, and Amisulpride for psychotic symptoms. Psychological (non-pharmacological) interventions mainly consisted in: supportive counseling, breathing exercises for anxious patients and sleep routines reframing for patients with insomnia.

\section{Assessing Consultation-Liaison Psychiatry for COVID-19 Inpatients}

To evaluate both the global need for liaison psychiatry and the characteristics of COVID-19 inpatients who benefit from psychiatric interventions, we performed a retrospective chart review from March 29th to April 24th. We started our study a few weeks after the first case of COVID-19 was diagnosed in the country.

Results Up to April 24, 2020, 144 patients were admitted with a confirmed diagnosis of COVID-19, and 36 (25\%) of them benefited from psychiatric consultation. Among those 36 patients, 20 (55.5\%) were women, 29 (80.5\%) had non-severe infection, their mean age was 40.3 years (min-max:19-85).

Among patients who received psychiatric intervention, 29 $(80.5 \%)$ had no previous psychiatric history. Of these patients, $24(66.66 \%)$ showed anxiety symptoms and $21(58.3 \%)$ had insomnia. Moreover, $10(27.7 \%)$ patients lost one of their relatives, from COVID-19, while hospitalized, and 9 of them showed distress related to bereavement. While all these patients were undergoing hydroxychloroquine treatment, 2 $(5,5 \%)$ of them experienced psychiatric side effects: panic attack in one patient, and manic episode with psychotic symptoms in the other. In total, we prescribed psychotropic medications for $20(55,5 \%)$ patients that consisted of anxiolytics/ hypnotics for 19 of them, and antipsychotic for one patient.

\section{Discussion}

Assessing and monitoring vulnerable groups during COVID19 outbreak, and finding the best way to deliver mental health services for them, was designated as an immediate target by an interdisciplinary group of 24 world-leading experts from across the bio-psycho-social spectrum of expertise in mental health science in March and April 2020 (Holmes et al., 2020). Our strategy allowed shaping a unique « home-made » circuit for COVID-19 inpatients. Indeed, in our sample, one of four patients needed psychiatric evaluation and intervention. Thus, psychiatry ranked second after cardiology in terms of requested expert advice for COVID-19 patients. Anxiety and insomnia are the two main symptoms expressed by patients of our sample. This finding confirms what has been described among inpatients in China (Hu et al., 2020; Wang et al., 2020), where depression, anxiety and insomnia were the most reported psychological disturbances; but also among the general population in Germany (Fatke, Hölzle, Frank, \& Förstl, 2020), China (Li et al., 2020) and Italy (De Girolamo et al., 2020), during the COVID-19 outbreak. Concerning the prescription of anxiolytics and hypnotics, it was parsimonious, at minimal dosages, using molecules with a short half-life, and after eliminating the contraindications, in particular the respiratory ones. One third of our patients who received psychiatric evaluation and intervention, were in bereavement-related distress after losing a relative who succumbed to COVID-19. Being in bereavement while hospitalized is particularly difficult: bereaved inpatients are denied the opportunity to see their deceased relative for the last time, to be with their families, or to attend the funeral rites. These exceptional circumstances may disrupt the normal mourning process and expose bereaved inpatients with COVID-19 to complicated grief (Simon, 2013). Other factors may be involved in the occurrence of neuropsychiatric sequelae in COVID-19 patients who received treatment in the hospital isolation wards, such as the emotions experienced by patients after being infected, external factors like discrimination and quality of medical care; and psychological factors like coping and self-help behavior (Hao et al., 2020).

Our study have some limitations: psychiatric evaluation did not include standardized questionnaires. Therefore, we could not quantify precisely the intensity of our patient's symptoms and evaluate with accuracy our interventions' efficiency. The relatively small sample size, and the monocentric nature of the study, do not allow our conclusions to be generalized to the entire population of similar patients, but the study provides insight into a liaison psychiatry experiment that can be adapted to suit other contexts.

\section{Conclusion}

In our experience, psychiatric interventions were one of the most requested for COVID-19 inpatients. Adapting liaison psychiatry to these special circumstances and for specific needs of this population, is one of the measures that may reduce the psychic impact of the pandemic on these patients. 
Acknowledgments - This research did not receive any specific grant from funding agencies in the public, commercial, or not-for-profit sectors.

- The datasets generated during and/or analysed during the current study are available from the corresponding author on reasonable request.

- Protocol was approved and validated by the Cheikh Khalifa International University Hospital ethical committee.

\section{Compliance with Ethical Standards}

Conflict of Interest Authors declare no conflict of interest.

\section{References}

Asdmunson, G. (2020). How health anxiety influences responses to viral outbreaks like COVID-19: What all decision makers, health authorities, and health care professionals need to know. Journal of Anxiety Disorders, 71, 102211. https://doi.org/10.1016/j.janxdis.2020. 102211.

De Girolamo, G., Cerveri, G., Clerici, M., Monzani, E., Spinogatti, F., Starace, F., Tura, G., \& Vita, A. (2020). Mental health in the coronavirus disease 2019 emergency-the Italian response. JAMA Psychiatry, 77(9), 974-976. https://doi.org/10.1001/ jamapsychiatry.2020.1276.

Fatke, B., Hölzle, P., Frank, A., \& Förstl, H. (2020). Psychische Probleme in der Pandemie - Beobachtungen während der COVID-19-Krise [COVID-19 Crisis: Early Observations on a Pandemic's Psychiatric Problems]. Deutsche Medizinische Wochenschrift, 145(10), 675681. German. https://doi.org/10.1055/a-1147-2889.

Hao, F., Tam, W., Hu, X., Tan, W., Jiang, L., Jiang, X., Zhang, L., Zhao, X., Zou, Y., Hu, Y., Luo, X., McIntyre, R. S., Quek, T., Tran, B. X., Zhang, Z., Pham, H. Q., Ho, C. S. H., \& Ho, R. C. M. (2020). A quantitative and qualitative study on the neuropsychiatric sequelae of acutely ill COVID-19 inpatients in isolation facilities.
Translational Psychiatry, 10(1), 355. https://doi.org/10.1038/ s41398-020-01039-2.

Holmes, E. A., O'Connor, R. C., Perry, V. H., Tracey, I., Wessely, S., Arseneault, L., ..., Ed Bullmore. (2020). Multidisciplinary research priorities for the COVID-19 pandemic: A call for action for mental health science. Lancet Psychiatry, 7, 547-560. https://doi.org/10. 1016/S2215-0366(20)30168-1.

https://www.who.int/publications-detail/WHO-2019-nCoVMentalHealth-2020.1 (n.d.). Accessed Nov 03.

Hu, Y., Chen, Y., Zheng, Y., You, C., Tan, J., Hu, L., Zhang, Z., \& Ding, L. (2020). Factors related to mental health of inpatients with COVID-19 in Wuhan, China. Brain, Behavior, and Immunity, 89, 587-593. https://doi.org/10.1016/j.bbi.2020.07.016.

Li, W., Yang, Y., Liu, Z.-H., Zhao, Y.-J., Zhang, Q., Zhang, L., Cheung, T., \& Xiang, Y.-T. (2020). Progression of mental health services during the COVID-19 outbreak in China. International Journal of Biological Sciences, 16(10), 1732-1738. https://doi.org/10.7150/ ijbs. 45120 .

Simon, N. M. (2013). Treating complicated grief. JAMA., 310(4), 416423. https://doi.org/10.1001/jama.2013.8614.

Wang, C., Pan, R., Wan, X., Tan, Y., Xu, L., Ho, C. S., \& Ho, R. C. (2020). Immediate psychological responses and associated factors during the initial stage of the 2019 Coronavirus disease (COVID-19) epidemic among the general population in China. International Journal of Environmental Research and Public Health, 17, 1729. https://doi.org/10.3390/ijerph17051729.

Wang, Y., Zhu, L. Y., Ma, Y. F., Bo, H. X., Deng, H. B., Cao, J., Wang, Y., Wang, X. J., Xu, Y., Lu, Q. D., Wang, H., \& Wu, X. J. (2020). Association of insomnia disorder with sociodemographic factors and poor mental health in COVID-19 inpatients in China. Sleep Medicine, 75, 282-286. https://doi.org/10.1016/j.sleep.2020.06. 011.

Publisher's Note Springer Nature remains neutral with regard to jurisdictional claims in published maps and institutional affiliations. 